\title{
Vulvar Adenocarcinoma
}

National Cancer Institute

\section{Source}

National Cancer Institute. Vulvar Adenocarcinoma. NCI Thesaurus. Code C6380.

An adenocarcinoma that arises from the vulva. Representative examples include

Bartholin gland adenocarcinoma, eccrine adenocarcinoma, apocrine adenocarcinoma, and sebaceous carcinoma. 